\title{
VIZUALIZATION OF THE CAVITATING JET USING FLASHLAMP FOR ILLUMINATION
}

\author{
Marek MLKVIK, Róbert OLŠIAK, Branislav KNÍŽAT
}

\begin{abstract}
The contribution deals with the ways of the illumination of the cavitating jet for the purposes of the visualization. The cavitation is induced by the fluid flow through the orifice with the diameter of $0,3 \mathrm{~mm}$. Region of the cavitating jet is illuminated by a different light sources. Goal of this paper is to compare the quality and the informative value of the obtained visual data. In addition, complicated structure of the cavitating jet is also shown and described
\end{abstract}

\section{INTRODUCTION}

The knowledge of the structure and the behavior of the cavitating jet is very important for the design and the optimalization of the devices working on the principle of cavitation. Cavitating jets are used in wide spectrum of the applications. For example cavitation is used for cleaning the paint and the dirt from the solid surfaces, removing of the explosives from ammunition [1], deep drilling [2]. Known are also the applications in the surgery, in cutting of the different materials or for killing the bacteria without using the chemicals. Because the cavitating jets are more and more used, the need to better understand of the behavior and structure of the cavitation raises. Commonly used criteria for description of the cavitation (like cavitation number, criteria for describing of the destructive effects) describes only the the effects on the solid surfaces. But they give no idea about the origin of these effects. For better design of the cavitating jets is important to better understand the behavior and structure of the cavitation dependent on other parameters (like cavitation number, pressures, velocity, destruction effect). For this purpose, the high-speed imagination can be used very effectively. And one of the key parameters in high-speed imagination of the cavitating jet is the illumination source (Figure 1).

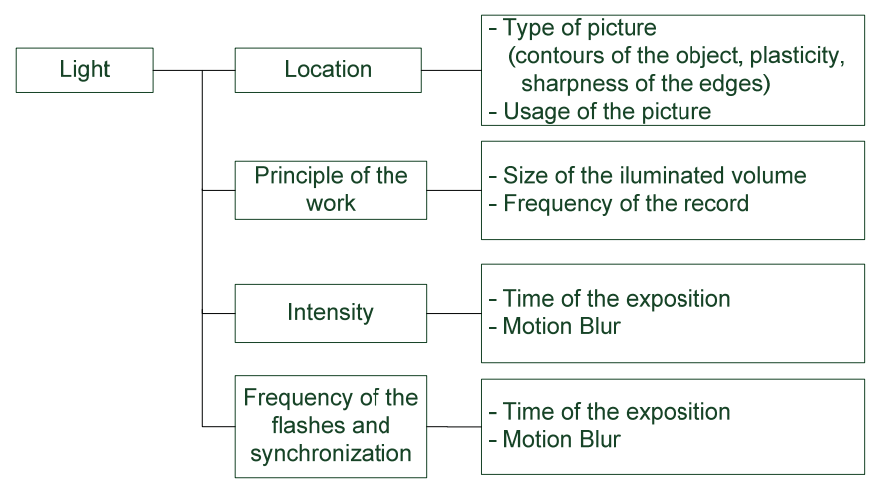

Figure 1: Function of the light in high-speed imagination

\footnotetext{
- Faculty of Mechanical Engineering, STU in bratislava, Institute of Process and Fluid Engineering, Nám. Slobody 17,812 31, Bratislava 1
}

This is an Open Access article distributed under the terms of the Creative Commons Attribution License 2.0, which permits unrestricted use, distribution, and reproduction in any medium, provided the original work is properly cited. 
The importance of the illumination will be demonstrated by the comparison of the pictures obtained by using different light sources. Structure and behavior of the cavitation jet will be also demonstrated by different regimes of the flow.

\section{DESCRIPTION OF THE MEASURING CIRCUIT}

The measuring circuit for the experiments with the cavitation consists of four main parts (Figure 2): hydraulic circuit, specimen, DAQ system and system used for visualization. All of the parts are synchronized by the industrial computer.

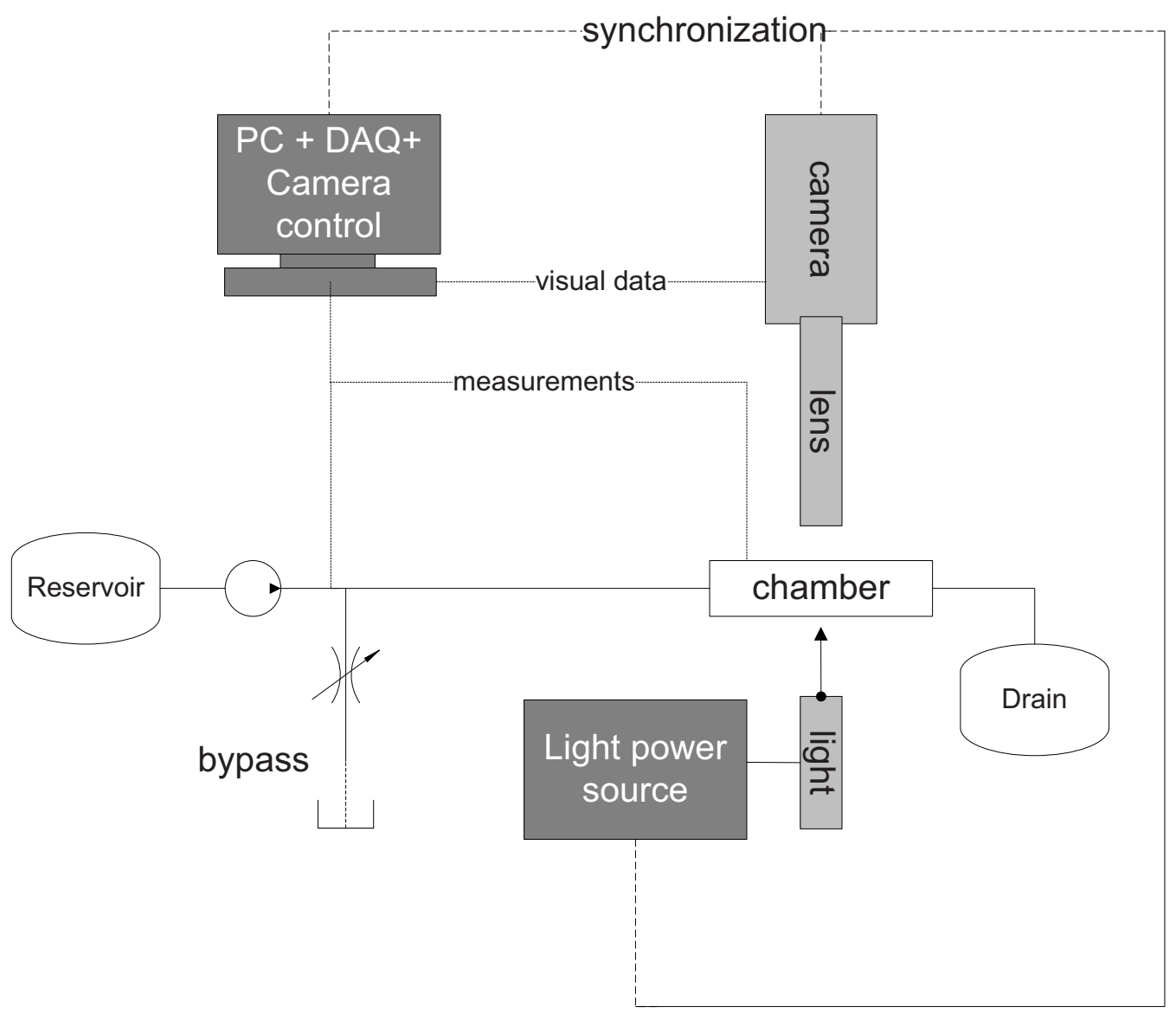

Figure 2: Scheme of the measuring circuit

Visualization part consist of the high speed camera (IDT redlake Y3), test chamber and the illumination. Optics of the camera consist from the telecentric microscopic objective and the mounting piece for the objective. Total visible area in these configuration is $3 \times 3 \mathrm{~mm}$.

\section{ILLUMINATION OF THE TEST CHAMBER}

Continual illumination source SCHOTT KL-1500 and the pulse flashlight NANOLITE KL-L with power source MINISTROBOKIN were used for the experiments. Description of the devices is in Tab 1 and Tab 2. 


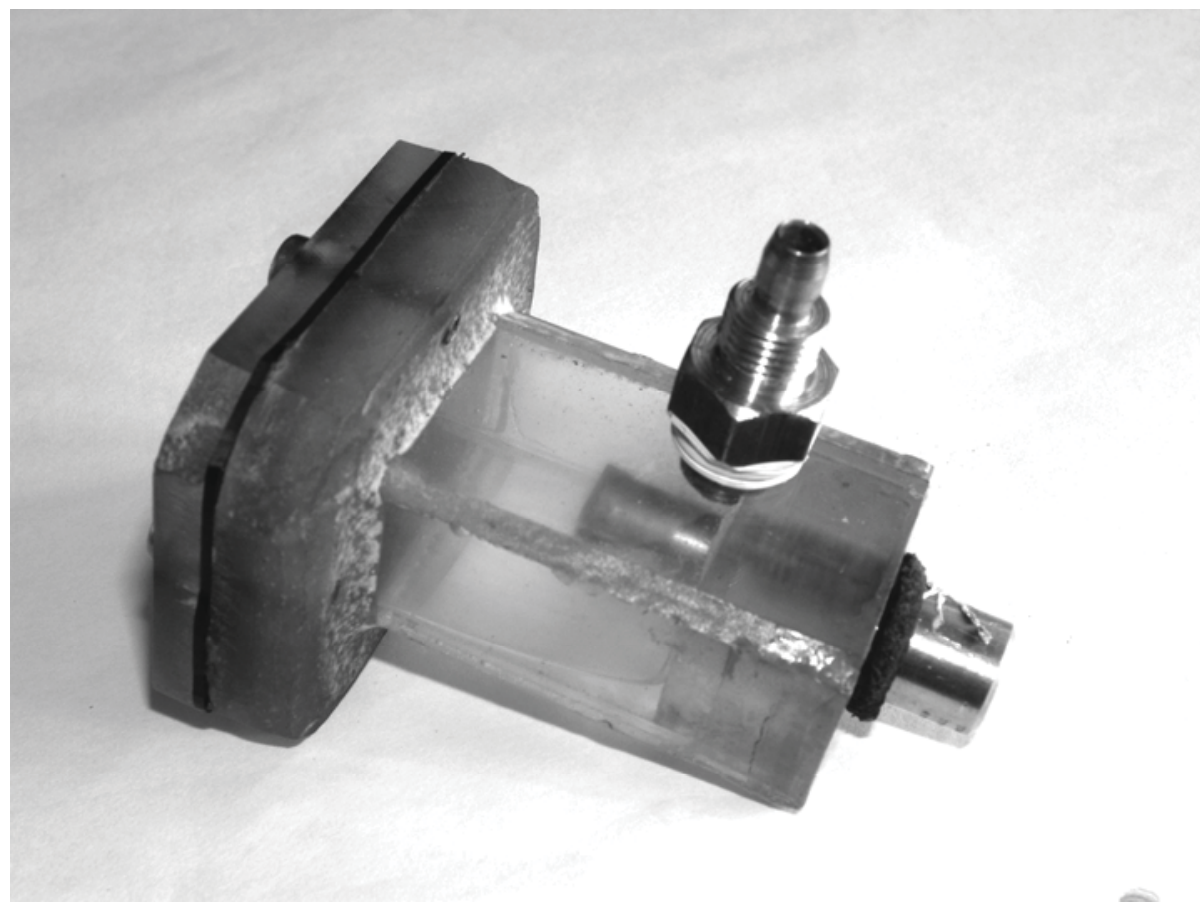

Figure 3: Test chamber

Tab 1 Properties of used flash light source

\begin{tabular}{|l|l|}
\hline Principle of work & Electric discharge \\
\hline Type of the light & White, cold \\
\hline Maximum frequency & $20000 \mathrm{~Hz}$ \\
\hline Flash duration & $18 \mathrm{~ns}$ \\
\hline Energy of the flash & $25 \mathrm{~mJ}$ \\
\hline $\begin{array}{l}\text { Maxminum ammount of flashes by max. } \\
\text { frequency }\end{array}$ & 200 \\
\hline Synchronization & External or internal \\
\hline Used focusing optics & no \\
\hline
\end{tabular}

Tab 2 Properties of used continual light source

\begin{tabular}{|l|l|}
\hline Type of the light & White, range cold to warm \\
\hline Power & $150 \mathrm{~W}$ \\
\hline Synchronization & None \\
\hline Used focusing optics & Optical fibre \\
\hline
\end{tabular}

With the flashlamp, the pictures were exposed using the shadow illumination. This type of the illumination was selected because of better visibility and sharpness of the corners of the cavitating jet. Also the tiny separated bubbles near main jet are better visible using this type of illumination. No additive optics is installed in the flashlamp, so no other directions of illumination are possible in this configuration.

Used laboratory illumination source gives continual white light with optional temperature. As focusing optics is used optical fibre. Direction of illumination can be changed. 


\section{Observations}

On the Figure 4 can be seen the cavitation jet in different regimes. The pictures were obtained using the flashlamp.
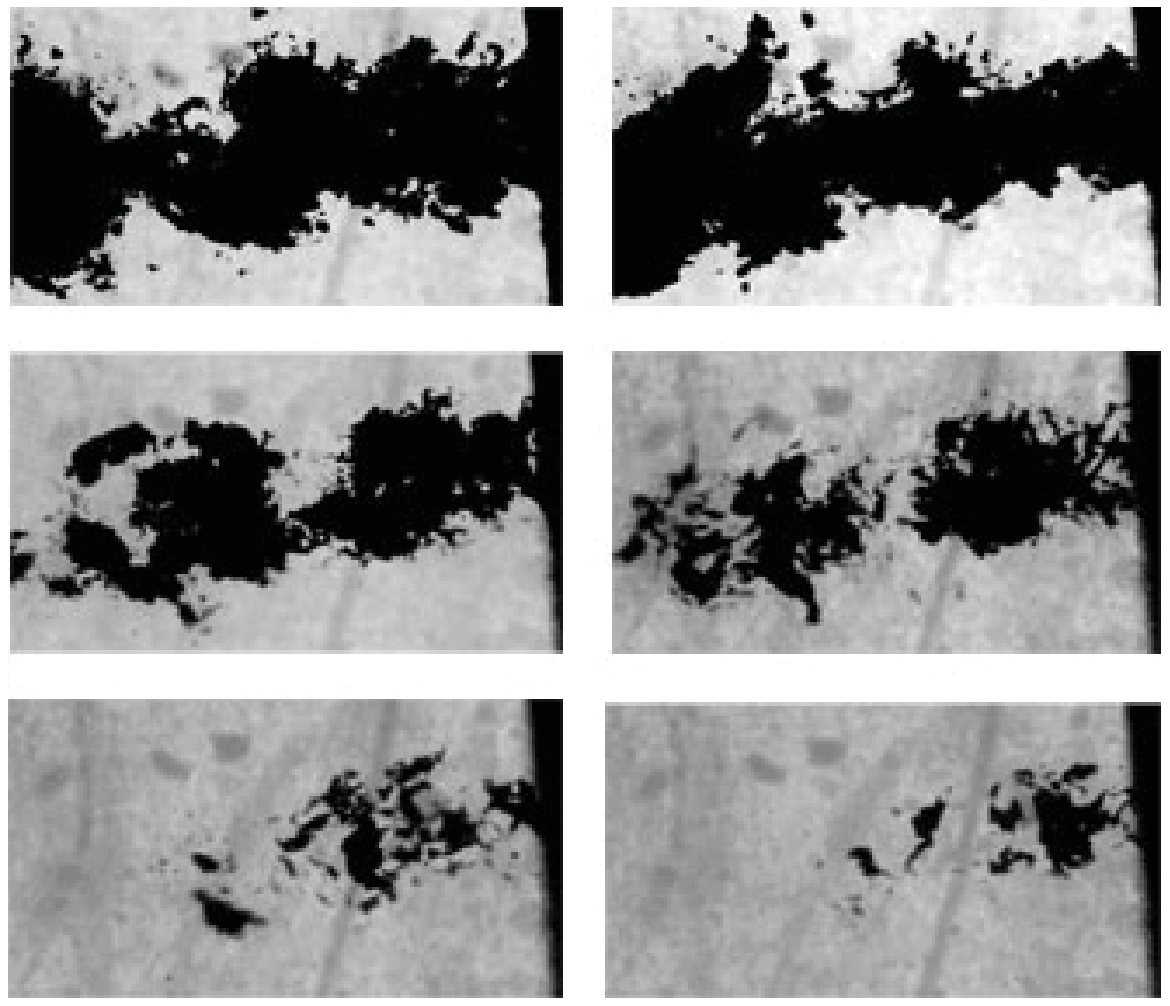

FLOW DIRECTION

Figure 4: Structure of the cavitation cloud in flow with diferent cavitation number, exposure time $=18 \mathrm{~ns},(\sigma=0.040, \sigma=0.035, \sigma=0.058, \sigma=0.066$, $\sigma=0.098, \sigma=0.125$ )

Complicated structure of the cavitating jet can be observed on the pictures (Figure 4). It can be seen that even by very low cavitation numbers the jet isn't symmetric and has a very complicated shape a changes very fast. This observations are in accordance with $[3],[4]$. Using of the very short exposition time (18 ns) allows detailed exploration of the structure and behavior of the cavitating jet.

Pictures obtained using continual light source (Figure 5) also shows the structure of the jet. In this case, the exposure is $2 \mu \mathrm{s}$. This is caused by low intensity of the light. A strong motion blur can be observed on this pictures, so the pictures don't have the informative value so high as pictures obtained with flashlamp.

Absolutely unsuitable is combination of the continual light and other type of the illumination as shadow illumination. As can be seen on the Figure 6, it is impossible to find sharp corners of the jet or find the structure. The motion blur is very strong, so the jet looks like homogenous object without any complicated structure. Only possibility to use this type of illumination is to use the more intensive light source, so shorter exposure time can be used. 

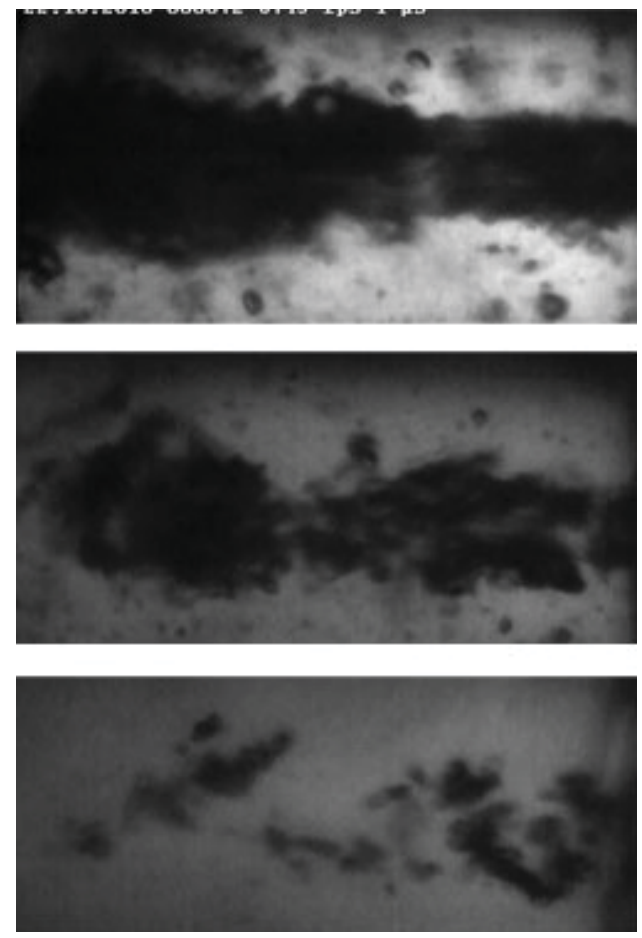

FLOW DIRECTION

Figure 5: Structure of the cavitation cloud captured with continual light by different cavitation number, exposure time $=1 \mu s,(\sigma=0.040, \sigma=0.066, \sigma=0.125)$

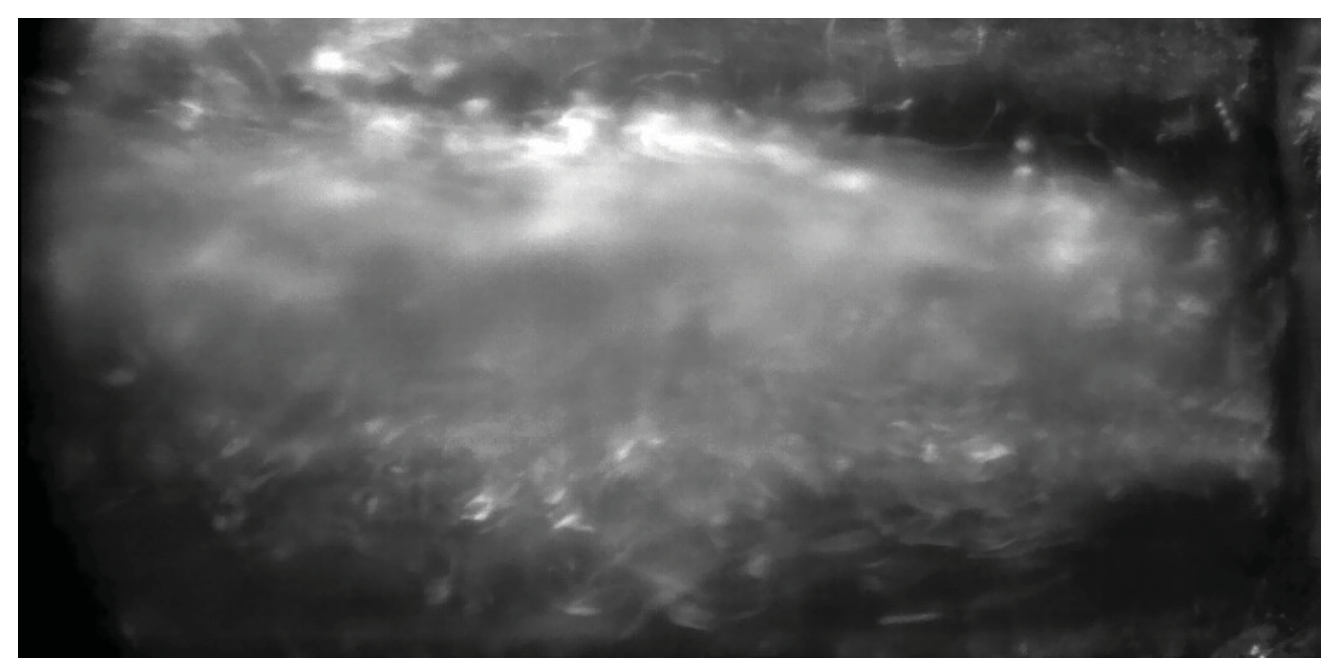

$\longrightarrow$ FLOW DIRECTION

Figure 6: Structure of the cavitation cloud captured with continual light $(\sigma=0.040)$, exposure time $40 \mu s$ 


\section{Conclusion}

Observing the cavitation without using the high-speed imagination and the powerful enough illumination source can give us idea that cavitation jet is homogenous, symmetric and stable area containing cavitation bubbles. High-speed camera show us that the jet is very complicated, not homogenous structure containing a lot of the substructures and it is changing very fast in time. This non-stationary behavior of the jet has strong influence on the destructive effect of the cavitation. Better understanding of this behavior is essential for next development and design of cavitation jets.

To obtain the usable visual data, with the sufficient quality for research, is essential to use powerful visualization hardware. The light is very important part of this hardware. Contribution clearly demonstrates the influence of the illumination to the quality and the informative value of the obtained visual data. Clearly, in our case, ist usage of the flashlight is best way to illuminate cavitating jet. It's caused by a very high energy of the single flashes. This allows to use very short exposition time, which is critical factor in imagination of high-speed phenomenon such as cavitation. Using of exposition time in order of nanoseconds give us way to see structure of the jet without motion blur. Even tiny separated bubbles can be seen. Tracking of these bubbles gives us only way to calculate the flowfield near the cavitating jet. It's because inserting of any other particles to the examined fluid can change the conditions of the experiment.

\section{ACKNOWLEDgment}

THIS WORK WAS SUPPORTED BY A SCIENTIFIC GRANT AGENCY VEGA UNDER CONTRACT NUMBER 1/0251/11

\section{REFERENCES}

[1] Summers David A., Worsey Paul N.: The use of high pressure water jets to wash out explosives, Proc. 6th Int. Conf. on Erosion by Liquid and Solid Impact, 1988

[2] Kollé J.J.: A comparison of water jet, abrasive jet and rotary diamond drilling in hard rock, Tempress Technologies Inc. 1999

[3] Hutli E.A.: Nedeljkovic M.,. Ilic V.: Visualization of Submerged Cavitating Jet: Part One, 16th Australasian Fluid Mechanics Conference, Australia 2007

[4] Soyama H., Yanuachi Y., Sato K., Ikohagi T. Oba R., Oshima R.: High speed observation of ultrahigh-speed submerged water jets, Experimental Thermal and Fluid Science 1996 